Draft VERSION OCTOBER 1, 2018

Preprint typeset using LATEX style AASTeX6 v. 1.0

\title{
SENSITIVITY OF THE CHERENKOV TELESCOPE ARRAY TO THE DETECTION OF INTERGALACTIC MAGNETIC FIELDS
}

\author{
Manuel Meyer and Jan Conrad \\ The Oskar Klein Center for CosmoParticle Physics, Department of Physics, Stockholm University, Albanova, SE-10691 Stockholm, \\ Sweden
}

Hugh DiCkinson

Department of Physics \& Astronomy, Iowa State University, Ames, IA 50011-3160, USA

\begin{abstract}
Very high energy (VHE; energy $E \gtrsim 100 \mathrm{GeV}$ ) $\gamma$-rays originating from extragalactic sources undergo pair production with low-energy photons of background radiation fields. These pairs can inverseCompton-scatter background photons, initiating an electromagnetic cascade. The spatial and temporal structure of this secondary $\gamma$-ray signal is altered as the $e^{+} e^{-}$pairs are deflected in an intergalactic magnetic field (IGMF). We investigate how VHE observations with the future Cherenkov Telescope Array, with its high angular resolution and broad energy range, can potentially probe the IGMF. We identify promising sources and simulate $\gamma$-ray spectra over a wide range of values of the IGMF strength and coherence length using the publicly available ELMAG Monte Carlo code. Combining simulated observations in a joint likelihood approach, we find that current limits on the IGMF can be significantly improved. The projected sensitivity depends strongly on the time a source has been $\gamma$-ray active and on the emitted maximum $\gamma$-ray energy.
\end{abstract}

Keywords: astroparticle physics - magnetic fields - BL Lacertae objects: general - gamma-rays: galaxies

\section{INTRODUCTION}

The origin of the magnetic fields ubiquitously present in galaxies, galaxy clusters, and perhaps filaments of large-scale structure is yet unknown. The general consensus is that observed fields can (at least partially) be explained by pre-existing fields that are amplified during the gravitational collapse of forming structures via flux compression and dynamos (see, e.g., Widrow 2002; Kulsrud \& Zweibel 2008; Durrer \& Neronov 2013, for reviews). However, little is known about the required seed fields. On the one hand, they could have formed in the very early Universe during the electroweak or QCD phase transition (e.g., Grasso \& Rubinstein 2001; Widrow 2002) or during inflation (e.g., Durrer \& Neronov 2013). On the other hand, the seed fields could be of astrophysical origin and could have been produced during the formation of large-scale structures at redshifts $z \lesssim 10$. The voids could have been polluted by

manuel.meyer@fysik.su.se

conrad@fysik.su.se

hughd@iastate.edu magnetic fields through galactic outflows caused by star formation (Bertone et al. 2006) or active galactic nuclei (AGNs; Rees 1987; Daly \& Loeb 1990; Ensslin et al. 1997; Furlanetto \& Loeb 2001). The two scenarios could be discerned by measuring the strength of intergalactic magnetic fields (IGMFs) in voids, $B$, and their coherence length $\lambda$ simultaneously. The coherence lengths of primordial fields should be $\lambda \lesssim \mathrm{kpc}$, whereas astrophysical fields should have field strengths $B \lesssim 10^{-9} \mathrm{G}$ with $\lambda \gtrsim \operatorname{kpc}$ (Durrer \& Neronov 2013).

So far, no direct measurements of $B$ and $\lambda$ exist. The nonobservation of Faraday rotation induced by an IGMF in quasar observations leads to upper limits of $B \lesssim 10^{-9} \mathrm{G}$ for megaparsec-scale coherence lengths (Blasi et al. 1999; Pshirkov et al. 2015). If the IGMF is of primordial origin, it will affect the primordial plasma and will leave specific imprints on the spectrum and polarization of the cosmic microwave background (CMB). Planck observations yield limits on the primordial IGMF with $B \lesssim 10^{-9} \mathrm{G}$ for $\lambda=1 \mathrm{Mpc}$, where the exact value depends on the considered IGMF model (Ade et al. 2015). Simulations of the formation of galaxy clusters and the propagation of ultrahigh energy cosmic rays sug- 
gest lower values of the order of $B \lesssim 10^{-12} \mathrm{G}$ (Sigl et al. 2004; Dolag et al. 2005).

Observation of $\gamma$-rays originating from blazars, AGNs with their jet closely aligned along the line of sight to the observer, provide an independent probe of the IGMF. The very high energy (VHE; energy $E \gtrsim 100 \mathrm{GeV}$ ) flux of AGN is attenuated due to the interaction of $\gamma$ rays with photons of the extragalactic background light (EBL), $\gamma+\gamma_{\mathrm{EBL}} \rightarrow e^{+}+e^{-}$(Nikishov 1962; Jelley 1966; Gould \& Schréder 1967b,a; Dwek \& Krennrich 2013). The attenuation scales exponentially with the optical depth $\tau(E, z)$, a monotonically increasing function with both the primary $\gamma$-ray energy $E$ and the source redshift $z$. The produced pairs can inverse-Compton (IC) scatter photons of the CMB and EBL and induce an electromagnetic cascade (Protheroe \& Stanev 1993). As the pairs are deflected in the magnetic field, the angular and time structure of the secondary photon signal depends on the strength and morphology of the IGMF. Under the assumption of a certain EBL model and intrinsic source spectrum, the nonobservation of the cascade component at $\mathrm{GeV}$ energies with the Fermi Large Area Telescope (LAT) led to a lower limit of $B \gtrsim 10^{-16} \mathrm{G}$ for $\lambda=1 \mathrm{Mpc}$ (Neronov \& Vovk 2010; Tavecchio et al. 2011), or conversely a lower limit on the the filling factor of the IGMF along the line of sight (Dolag et al. 2011). Additionally, the IGMF induces a time delay of the cascade emission compared to the primary source emission (Plaga 1995; Dai et al. 2002; Murase et al. 2008; Takahashi et al. 2008; Neronov \& Semikoz 2009). If this is taken into account, together with conservative assumptions on the AGN $\gamma$-ray activity, the limit is relaxed by several orders of magnitude, $B \gtrsim 10^{-19} \mathrm{G}$, as derived from semianalytical models (Dermer et al. 2011; Huan et al. 2011; Finke et al. 2015) and full Monte Carlo simulations for simultaneous observations with imaging air Cherenkov telescopes (IACTs) and the Fermi LAT, leading to $B \gtrsim 10^{-17} \mathrm{G}$ (Taylor et al. 2011). For small EBL photon densities and taking uncertainties of the intrinsic source spectrum into account, the hypothesis of a zero IGMF cannot be rejected (Arlen et al. 2014).

Strong magnetic fields could also be detected through the angular profile of the $\gamma$-ray emission since the $e^{+} e^{-}$ pairs would be quickly isotropized and extended $\gamma$-ray halos would form around sources (Aharonian et al. 1994; Dolag et al. 2009; Elyiv et al. 2009; Neronov \& Semikoz 2009). No extended emission was found in H.E.S.S. observations (Abramowski et al. 2014), whereas indications for pair halos were found in Fermi-LAT data, suggesting magnetic fields of the order of $10^{-17} \mathrm{G} \lesssim B \lesssim 10^{-15} \mathrm{G}$ for $\lambda=1 \mathrm{Mpc}$ (Chen et al. 2015a).

A helical IGMF could be detected through parity odd signatures of the arrival directions of $\gamma$-rays produced in the electromagnetic cascade (Tashiro \& Vachaspati
2013). An analysis of the diffuse $\gamma$-ray background observed with Fermi LAT indeed suggests such correlations with favored magnetic fields $B \sim 5 \times 10^{-14} \mathrm{G}$ ordered over $10 \mathrm{Mpc}$ scales (Chen et al. 2015b).

The cascade could be suppressed if the $e^{+} e^{-}$pairs could lose their energy primarily via plasma instabilities instead of IC scattering (Broderick et al. 2012). Particlein-cell simulations suggest that the energy loss due to the instabilities plays only a subdominant role (Sironi \& Giannios 2014). However, as noted by Menzler \& Schlickeiser (2015), these simulations require extrapolations over many orders of magnitude in the density ratio between the beam and the background plasma. The authors find instead a reduction of the cascade flux by a factor of 0.1 for typical blazars.

Assuming no energy losses in plasma instabilities, we investigate the prospects of the future Cherenkov Telescope Array (CTA) to detect the secondary cascade emission. The amount of cascade emission that arrives within a certain maximum delay time and within the CTA point spread function (PSF) depends on the IGMF, thereby allowing constraints on its strength (e.g. Taylor et al. 2011). We follow a similar approach here.

CTA will be composed of IACTs of different sizes, covering a large energy range between tens of $\mathrm{GeV}$ up to hundreds of $\mathrm{TeV}$ with an expected sensitivity improvement of a factor of 10 compared to currently operating IACTs (Actis et al. 2011). The energy resolution is envisaged to be of the order of $10 \%-15 \%$ and the spatial resolution of the order of arcminutes. The broad energy coverage makes it possible to detect the primary and secondary spectral components simultaneously. Throughout this paper, the "Array E" configuration of CTA will be assumed, which provides good sensitivity over the entire energy range (Bernlöhr et al. 2013). Current limits often rely on the combination of IACT and FermiLAT data, which are usually nonsimultaneous and suffer from the systematic uncertainty of potentially different energy scales. These issues are avoided with CTA observations.

The article is organized as follows. In Section 2 we present our source selection for promising blazars to search for the cascade. As described in Sec. tion 3, we use the publicly available ELMAG Monte Carlo code to generate spectra including a cascade for a wide range of IGMF morphologies. We use a standard likelihood ratio test to search for the cascade component (Section 4) and combine observations in a joint likelihood. We present our results in Section 5 before concluding in Section 6 .

\section{SOURCE SELECTION}

Promising targets to search for the cascade are blazars whose intrinsic emission extends to energies where the EBL absorption is strong. This requires sources with a 
hard intrinsic spectrum characterized by a simple power law without a cutoff, $\mathrm{d} N / \mathrm{d} E \propto E^{-\Gamma}$, with $\Gamma \lesssim 2$. In addition to being a high synchrotron peaked BL Lac $\left(\mathrm{HBL}, \log _{10}\left(\nu_{\mathrm{sync}} / \mathrm{Hz}\right)>15\right)$, promising sources also show a high ratio between the X-ray and radio flux, $F_{\mathrm{X}} / F_{\mathrm{R}} \gtrsim 10^{4}$, as well as an optical spectrum dominated by the host galaxy (Bonnoli et al. 2015). Bonnoli et al. used values for the X-ray and radio flux as listed in Plotkin et al. (2010), with $F_{\mathrm{X}}$ measured with ROSAT between 0.1 and $2.4 \mathrm{keV}$ and $F_{\mathrm{R}}=\nu F_{\nu}$ with $\nu=1.4 \mathrm{GHz}$. $1 \mathrm{ES} 0229+200$ is a typical example for such an "extreme" HBL (EHBL; Costamante et al. 2001).

We follow these requirements and select sources from the second Fermi catalog of hard sources detected above $50 \mathrm{GeV}$ (2FHL; Ackermann et al. 2016) that fulfill the following criteria:

1. Their redshift is known. Otherwise, it is not possible to determine the strength of the absorption.

2. They are HBLs, i.e. $\log _{10}\left(\nu_{\text {sync }} / \mathrm{Hz}\right)>15$.

3. They show a high ratio between their X-ray and radio flux, $F_{\mathrm{X}} / F_{\mathrm{R}}>10^{3}$, where the fluxes are taken from the third Fermi-LAT AGN catalog (3LAC; Ackermann et al. 2015). The X-ray flux is taken from the ROSAT all sky survey between 0.1 and $2.4 \mathrm{keV}$ and the the radio flux is determined from $F_{\mathrm{R}}=\nu F_{\nu}$. The frequency varies depending on the radio survey used (see Table 8 in the $3 \mathrm{LAC})$.

4. Integrating their observed $2 \mathrm{FHL}$ spectra between 1 and $2 \mathrm{TeV}$ should result in at least $1 \%$ of the integrated flux of the Crab Nebula in the same energy range (assuming the VHE Crab spectrum measured with H.E.S.S.; Aharonian et al. 2006).

5. The absorption-corrected spectra in the 2FHL (Domínguez \& Ajello 2015) follow power laws with an index $\Gamma \leqslant 1.7$. This value is chosen a posteriori, as softer spectra do not turn out to lead to a sizable flux of the cascade photons. By making this cut, we assume that the Fermi-LAT observations are not contaminated by the cascade. As we will see in Sec. 3, this is justified by taking the 2FHL error bars of most sources into account.

6 . The sources should show little $\gamma$-ray variability as we assume a steady $\gamma$-ray emission to calculate the cascade. Following Finke et al. (2015), we select sources with a variability index $<100$ as provided in the third Fermi source catalog (3FGL; Acero et al. 2015). This corresponds to a $4.8 \sigma$ significance that the source is variable.

7. They culminate at low zenith angles, $Z \leqslant 20^{\circ}$, in order to guarantee an energy threshold as low as possible. For this selection, we assume latitudes of $-24^{\circ}$ and $29^{\circ}$ for the southern and northern CTA site, respectively.

In addition to the above criteria, we demand that the cascade photons have an energy $>50 \mathrm{GeV}$. As the IC scattering with CMB photons with an average energy $\left\langle\epsilon_{\mathrm{CMB}}\right\rangle \approx 634 \mu \mathrm{eV}$ occurs entirely in the Thomson regime, the average energy of a cascade photon is

$$
\langle\epsilon\rangle=\frac{4}{3}\left\langle\epsilon_{\mathrm{CMB}}\right\rangle \gamma^{2} \approx 0.81\left(\frac{E}{\mathrm{TeV}}\right)^{2} \mathrm{GeV},
$$

with an electron Lorentz factor $\gamma=E / 2 m_{e} c^{2}$, where $m_{e}$ is the mass of the electron and $E$ the energy of the primary $\gamma$-ray. We estimate the maximum cascade photon energy from head-on IC scattering in the Thompson regime with a CMB photon with an energy of $\epsilon_{\mathrm{CMB}, 99} \approx 2 \mathrm{meV}$. The integral over the CMB photon density up to this energy is equal to $99 \%$ of the same integral between $[0 ; \infty)$. One finds a maximum cascade energy

$$
\epsilon_{\max }=4 \epsilon_{\mathrm{CMB}, 99} \gamma^{2} \approx 9\langle\epsilon\rangle .
$$

To decide whether $\epsilon_{\max }$ falls inside the CTA energy range, one has to make an assumption about the maximum energy of the primary $\gamma$-ray spectrum. A primary $\gamma$-ray spectrum that extends to high energies will also lead to more energy that can be reprocessed in the cascade. Evidence for emission at energies beyond $\tau>5$ has been found in several blazar observations, e.g. for 1ES 0229+200 (Aharonian et al. 2007), PKS 1424+240 (Archambault et al. 2014) with $z \geqslant 0.6035$ (Furniss et al. 2013), and PKS 0447-121 (Abramowski et al. 2013) assuming the redshift of $z=0.343 \pm 0.002$ (Muriel et al. 2015). We therefore assume that the spectrum extends to an energy where the optical depth $\tau=5$ (we will scrutinize this assumption in Sec. 5).

In total, nine HBLs listed in the 2FHL survive the applied cuts. From this list we further exclude the already TeV-detected sources IC 310, RBS 0413, RX J0648.7+1516, 1RXS J101015.9-311909, and B3 2247+381. None of the IACT spectra extend to high optical depths, and the measured indices are significantly softer than the ones listed in the 2FHL. The remaining four blazars are listed in Table 1 . We append $1 \mathrm{ES} 0229+200$ to the list, even though the source is not included in the $2 \mathrm{FHL}$.

\section{SIMULATIONS}

\subsection{Cascade Simulations}

The development of electromagnetic cascades in the intergalactic medium is simulated with the open-source Monte Carlo code ELMAG (for more details see Kachelrieß et al. 2012). ELMAG computes the resultant photon distribution based on initial prescriptions for the shape of 
Table 1. Sources selected for Simulation.

\begin{tabular}{lccccccccc}
\hline \hline Source name & $\begin{array}{c}\text { R.A. } \\
(\mathrm{deg})\end{array}$ & $\begin{array}{c}\text { Decl. } \\
(\mathrm{deg})\end{array}$ & $z$ & $\begin{array}{c}E_{\mathrm{HEP}} \\
(\mathrm{GeV})\end{array}$ & $\begin{array}{c}E_{\tau=5} \\
(\mathrm{TeV})\end{array}$ & $\log _{10}\left(\frac{\nu_{\mathrm{sync}}}{\mathrm{Hz}}\right)$ & $\begin{array}{c}F\left(10^{-10} \mathrm{ergs} \mathrm{cm}^{-2} \mathrm{~s}^{-1}\right) \\
\left(\text { Above } E_{\text {thr }} / \mathrm{TeV}\right)\end{array}$ & $\begin{array}{c}\Gamma \pm \sigma_{\Gamma} \\
F_{\mathrm{R}}\end{array}$ \\
\hline B2 0806+35 & 122.39 & 34.97 & 0.083 & 264.23 & 13.931 & 15.500 & $7.24(1.0)$ & $0.920 \pm 0.770$ & 3158 \\
PG 1218+304 & 185.34 & 30.16 & 0.182 & 513.20 & 6.488 & 16.590 & $2.43(0.2)$ & $1.630 \pm 0.270$ & 31508 \\
PMN J1548-2251 & 237.19 & -22.82 & 0.192 & 435.85 & 5.935 & 16.061 & $1.02(0.5)$ & $1.340 \pm 0.490$ & 15455 \\
1RXS J023832.6-311658 & 39.62 & -31.27 & 0.232 & 407.77 & 4.091 & 16.160 & $0.57(0.5)$ & $0.760 \pm 0.650$ & 9050 \\
\hline 1ES 0229+200 & 38.20 & 20.29 & 0.139 & - & 9.242 & 15.481 & $1.35(0.58)$ & 1.7 \\
\hline
\end{tabular}

Note. In addition to the source coordinates we give the redshift $z$ (the redshift of PMN J1548-2251 is taken from Shaw et al. 2013), the highest energy photon, $E_{\mathrm{HEP}}$, the energy at which $\tau=5, E_{\tau=5}$, the peak frequency of the synchrotron emission, the integrated energy flux $F$ between $E_{\mathrm{thr}}$ and $E_{\tau=5}$ assumed for the simulation, the spectral index of the intrinsic blazar spectrum $\Gamma$ with its uncertainty, and the $\mathrm{X}$-ray to radio flux ratio. X-ray and radio fluxes are taken from the $3 \mathrm{LAC}$. The values for the coordinates, $E_{\mathrm{HEP}}, \nu_{\mathrm{sync}}$, and $\Gamma$ (derived by de-absorbing the observed spectra with the EBL model of Domínguez et al. 2011) are taken from the 2FHL.

the intrinsic $\gamma$-ray spectrum, the spectrum and redshift evolution of the EBL, and the large-scale configuration of the IGMF. It adopts the simplifying assumption that the IGMF can be completely characterized by a universal intensity $B$ and a cell-like structure with coherence length $\lambda$. The scattering of the $e^{+} e^{-}$pairs on both EBL and CMB photons is taken into account. Photons with an energy above $\epsilon \geqslant \epsilon_{\text {thr }}$ are traced, where we set $\epsilon_{\mathrm{thr}}=1 \mathrm{GeV}$. Energy losses due to synchrotron radiation and IC scattering are integrated out for energies $<\epsilon_{\mathrm{thr}}$. We choose to trace all particles, i.e. setting $\alpha_{\text {sample }}=0$ (Kachelrieß et al. 2012).

Under the assumption that the $e^{+} e^{-}$pairs do not lose energy in plasma instabilities, we simulate the final photon distribution from $6 \times 10^{5}$ injected primary $\gamma$-rays with the following fiducial model assumptions for each considered source:

1. The intrinsic $\gamma$-ray spectrum is given by a power law, $\mathrm{d} N / \mathrm{d} E \propto E^{-\Gamma}$, with the absorptioncorrected index of the 2FHL (see Table 1). For 1ES $0229+200$, we assume an intrinsic index $\Gamma=$ 1.7 .

2. We choose the EBL model of Domínguez et al. (2011).

3 . We assume that the primary $\gamma$-ray emission extends to an energy for which $\tau_{\max }=5$. Beyond this energy, the emission is zero.

4. An opening angle of the blazar jet of $\theta_{\mathrm{j}}=6^{\circ}$ is assumed. In the approximation that the Doppler factor is equal to the bulk Lorentz factor $\Gamma_{\mathrm{L}}$ of the emitting plasma, this implies $\Gamma_{\mathrm{L}} \sim \theta_{\mathrm{j}}^{-1} \sim 10$.

5 . We simulate cascades for values of $(B, \lambda)$ on a $(9 \times 9)$ logarithmic spaced grid with $B_{\mathrm{G}}=$ $B / \mathrm{G} \in\left[10^{-19} ; 10^{-11}\right]$ and $\lambda_{\mathrm{Mpc}}=\lambda / \mathrm{Mpc} \in$ $\left[10^{-6} ; 10^{2}\right]$. These parameters cover the evidences for a nonzero IGMF (Chen et al. 2015a,b), as well as scenarios for astrophysical or primordial origins of the IGMF (e.g., Durrer \& Neronov 2013).

We discuss the impact of these assumptions in Sec. 5 .
ELMAG outputs the total observed spectrum $\epsilon F_{\epsilon}$ with primary and cascade emission binned in energy, angular separation $\delta \theta$, and time delay $\delta t$. Both $\delta \theta$ and $\delta t$ are due to the deflection of the $e^{+} e^{-}$pairs in the IGMF. We show an example of the output photon distribution as a function of energy and $\delta \theta(\delta t)$ for one pair of $(B, \lambda)$ values for 1 ES $0229+200$ in Figure 1. The distributions follow the theoretical expectations that $\delta \theta \propto \epsilon^{-1} B$ and $\delta t \propto \epsilon^{-5 / 2} B^{2}$ since the chosen value of $\lambda=1 \mathrm{Mpc} \gtrsim$ $D_{\text {IC }}$, where $D_{\text {IC }}$ is the IC cooling length (Neronov \& Semikoz 2009). With the Thomson cross section $\sigma_{T}$ and the energy density of the $\mathrm{CMB} u_{\mathrm{CMB}}=0.26 \mathrm{eV} \mathrm{cm}^{-3}$, the cooling length is

$$
D_{\mathrm{IC}}=\frac{3 m_{e} c^{2}}{4 \sigma_{\mathrm{T}} u_{\mathrm{CMB} \gamma}} \approx 0.7\left(\frac{E}{\mathrm{TeV}}\right)^{-1} \mathrm{Mpc} .
$$

For a cell-like IGMF, one expects $\delta \theta \propto \epsilon^{-3 / 4} B \sqrt{\lambda}$ and $\delta t \propto \epsilon^{-5 / 2} B^{2} \lambda$ for $\lambda \ll D_{\text {IC }}$.

\subsection{CTA Simulations}

We generate CTA observations by folding the ELMAG output spectra with the CTA instrumental response function (IRF) for the "Array E" configuration. The IRF and the expected background rate have been determined from Monte Carlo simulations (Bernlöhr et al. 2013). For each source (except $1 \mathrm{ES} 0229+200$ ), we simulate a $\mathrm{T}_{\mathrm{obs}}=20$ hours observation under a constant zenith angle of $20^{\circ}$ and a ratio between source and offsource exposure of $\alpha=0.2$. AGN observations of this duration are envisaged during the initial years of data taking (CTA Consortium 2016, in preparation). Since we assume that $1 \mathrm{ES} 0229+200$ has the softest intrinsic spectrum of all considered sources, the observation time is doubled to $40 \mathrm{hr}$.

We approximate the CTA PSF as a Heavyside step function that is nonzero within its $80 \%$ containment radius $r_{80}$. Hence, we discard all ELMAG output photons with $\delta \theta>r_{80}$ (indicated by the red solid line in the top panel of Figure 1). Furthermore, we assume that the sources have been active for $10 \mathrm{yr}$, approximately the time that $\gamma$-ray sources have been observed (red 


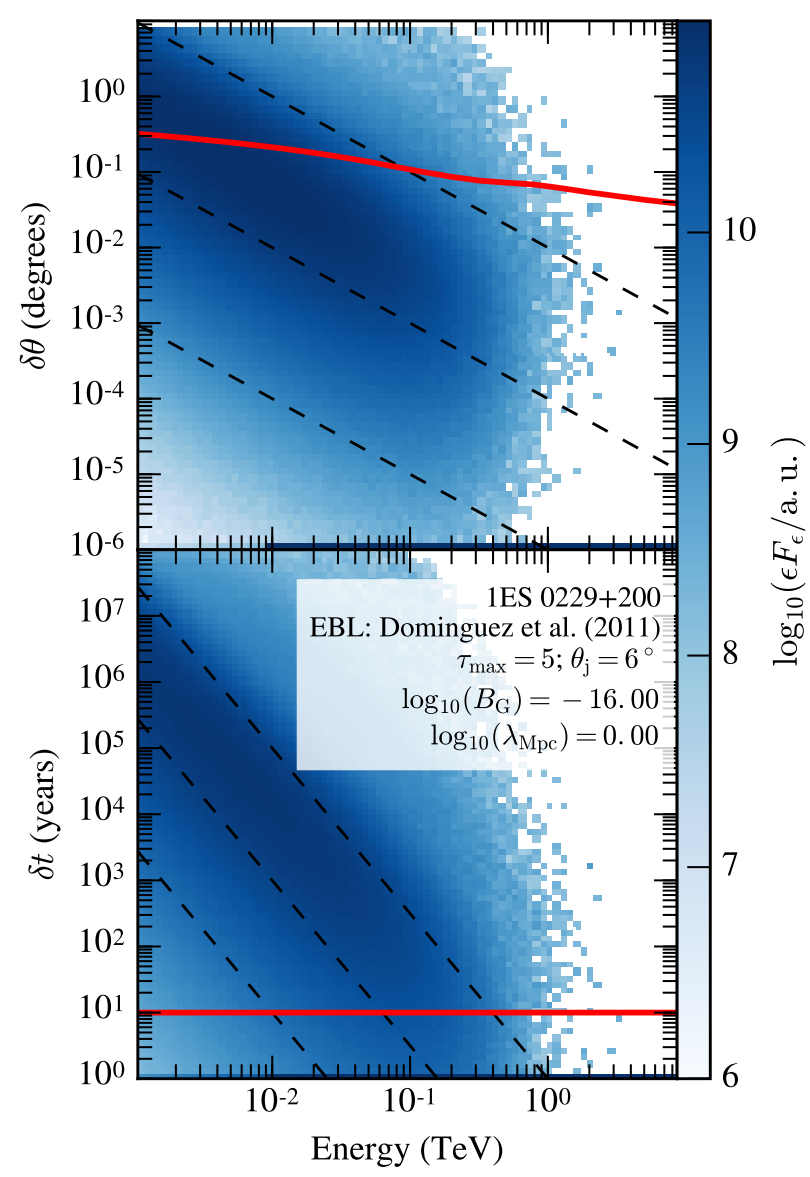

Figure 1. Two-dimensional histograms showing the output of the ELMAG Monte Carlo simulation. For the simulation, we assume the spectrum of $1 \mathrm{ES} 0229+200, B_{\mathrm{G}}=10^{-16}$, and $\lambda_{\mathrm{Mpc}}=1$. All other parameters are set to their fiducial values. The ELMAG output is binned in energy and $\delta \theta$ (top) or $\delta t$ (bottom) and each bin shows the contained $\epsilon F_{\epsilon}$ in arbitrary units (a.u.). To obtain the energy spectra (see Figure 3 ) within the PSF containment radius (or maximum time delay), one has to sum the histogram along the $\delta \theta(\delta t)$ axis for all entries below the red solid lines. Theoretical expectations for the energy dependence of $\delta \theta$ and $\delta t$ are shown as black dashed lines to guide the eye.

solid line in the bottom panel of Figure 1). This cut on the delay time effectively supersedes the PSF cut, since photons arriving with $\delta \theta>r_{80}$ usually have delay times $\delta t \gg 10 \mathrm{yr}$. We have verified this with the ELMAG simulations for all considered sources and three configurations of the magnetic field discussed in Sec. 5 . In Section 5, we also examine the impact on the results if the cut on the delay time is relaxed. The remaining fraction of the ELMAG output spectra is then interpolated with a cubic spline in order to guarantee a smooth spectrum for the CTA simulation. The spectra are rescaled, so that the integrated flux above a certain energy threshold $E_{\text {thr }}>\epsilon_{\max }$ is independent of $(B, \lambda)$. The assumed integrated fluxes and val- ues for $E_{\text {thr }}$ are listed in Table 1. For PG 1218+304, the integrated flux and $E_{\text {thr }}$ are chosen to match the VERITAS observation of this source (Madhavan, for the VERITAS Collaboration 2013). For 1ES 0229+200 we chose the values obtained with H.E.S.S. observations (Aharonian et al. 2007). For PMN J1548-2251 and 1RXS 023832.6-311658 we assume values similar to $1 \mathrm{ES} 0229+200$, namely, that the integrated flux above $500 \mathrm{GeV}$ is equal to $2 \%$ of the integrated flux of the Crab Nebula (C.U.), whereas for B2 $0806+35$ we take $F=5 \%$ C.U. above $1 \mathrm{TeV}$ (the Crab nebula spectrum is taken from Aharonian et al. 2006). All the assumed values are compatible with the results from the $2 \mathrm{FHL}$ except for B2 $0806+35$ and an IGMF close to zero. In this case, the cascade emission is also in mild tension with the flux upper limit obtained from VERITAS observations (Archambault et al. 2016).

We further note that the extrapolation of the intrinsic spectra up to $E_{\tau=5}$ yields luminosities $L$ that are consistent with the requirement that the radiation power $P$ of the jet is less than half the Eddington luminosity $P \sim L / 4 \Gamma_{\mathrm{L}}^{2} \leqslant L_{\text {edd }} / 2$ (Bonnoli et al. 2011), where we again approximated $\delta_{\mathrm{D}} \sim \Gamma_{\mathrm{L}}$. Following Meyer et al. (2012), we derive the luminosity by integrating the intrinsic spectra between $50 \mathrm{GeV}$ and $E_{\tau=5}$ and multiply the integral by $(1-z)^{2-\Gamma} \times 4 \pi d_{L}^{2}$. The first factor accounts for the $K$-correction and $d_{L}$ is the luminosity distance. Generically assuming black hole masses of $10^{8.5} M_{\odot}$, we find that $P$ is at most $\sim 0.2 \%$ of $L_{\text {edd }}$ for PG $1218+304$.

Following Meyer \& Conrad (2014), the rescaled and interpolated spectra are folded with the IRF and multiplied with the observation time to yield the number of expected counts for each source in energy bin $i, \mu_{i}$. The number of background events $b_{i}$ is obtained by multiplying the background rate derived from Monte Carlo simulations by $\mathrm{T}_{\mathrm{obs}}$. Adjacent energy bins in which the source is detected with a significance $S_{i}<2 \sigma$ are combined into one bin (the significance is evaluated with Eq. (17) of $\mathrm{Li} \& \mathrm{Ma}$ 1983). If the significance of the combined bin is still below $2 \sigma$, the bin is discarded. We show examples of the rescaled ELMAG spectra and the CTA simulation for all sources and three values of $B$ in Figure 2. For most sources, the 2FHL measurements are consistent with the CTA simulations even if the cascade excess is present.

\section{ANALYSIS METHOD}

Higher values of the IGMF strength and coherence length will lead to a stronger deflection of the $e^{+} e^{-}$pairs and cause larger time delays and angular separations of the cascade photons. With our chosen cuts on $\delta t$ and $\delta \theta$ this implies a diminished cascade flux. We use a Poisson likelihood ratio test to determine the compatibility 

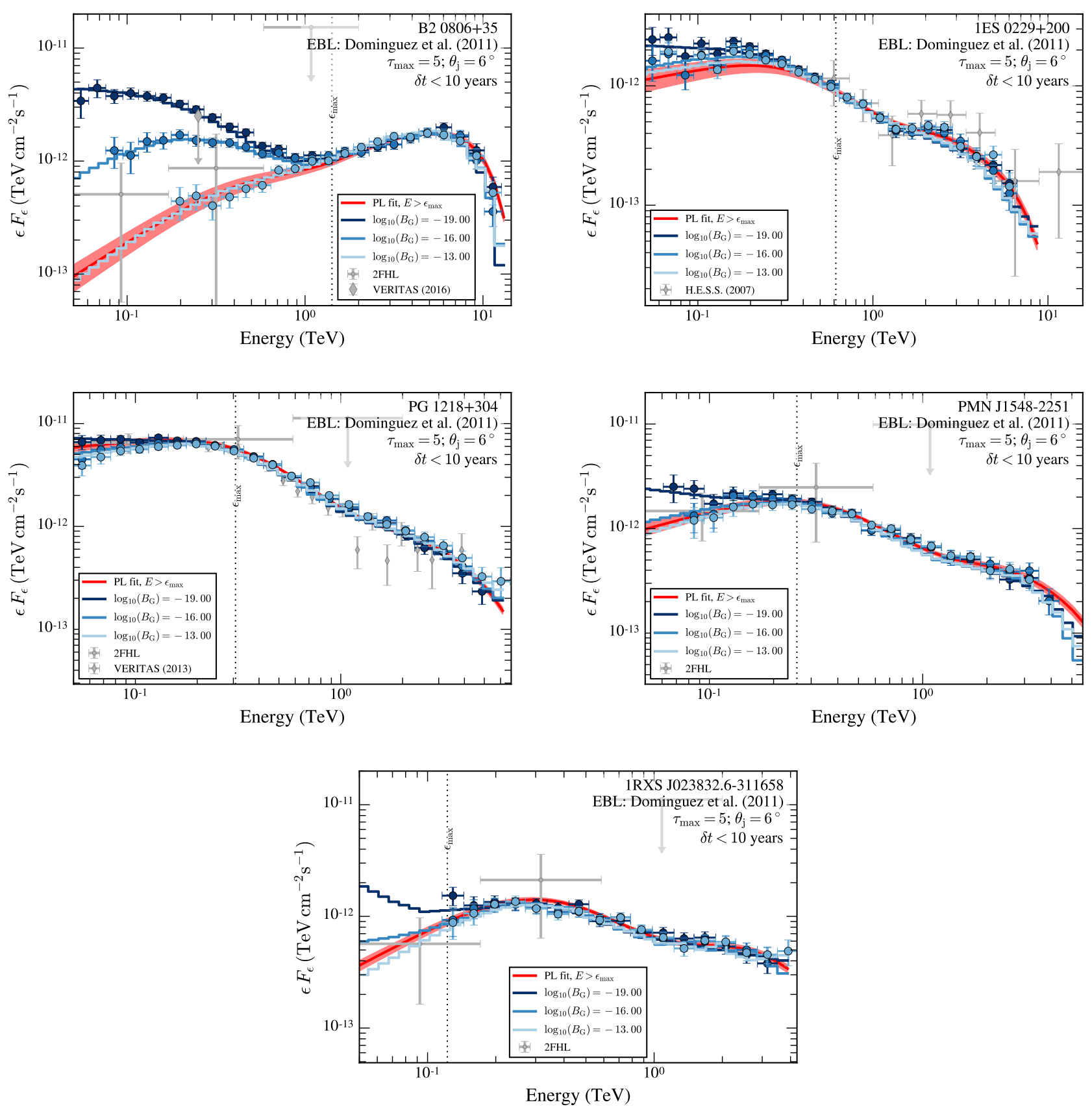

Figure 2. Simulated spectra for the selected sources assuming different magnetic field strengths and a constant coherence length of $\lambda_{\mathrm{Mpc}}=1$. In addition to the simulated CTA data (blue bullets), we show data points of the 2FHL and IACT observations where available (gray diamonds and squares). We apply both the time and angular separation cut to the simulated cascade (blue solid lines). The maximum cascade energy $\epsilon_{\max }$ is shown as a black dotted vertical line. A $\chi^{2}$ power-law (PL) fit including EBL absorption to the simulated data with $\log _{10}\left(B_{\mathrm{G}}\right)=-19$ and energy bins $E_{i}>\epsilon_{\max }$ is shown with red solid lines.

of a magnetic field hypothesis characterized through the expected number of counts $\mu$ for one set of values $(B, \lambda)$ with mock data $D$, generated under the same or a different hypothesis. For expected signal counts $\mu_{i}$ and background counts $b_{i}$ in the $i$ th energy bin, the likelihood of observing $x_{i}$ counts from the sky region including a source and $y_{i}$ counts from a background region is

$$
\mathcal{L}\left(\mu_{i}, b_{i} ; \alpha \mid x_{i}, y_{i}\right)=\operatorname{Pois}\left(x_{i} \mid \mu_{i}+b_{i}\right) \operatorname{Pois}\left(y_{i} \mid b_{i} / \alpha\right) .
$$

We only consider energy bins for which we expect a contribution from the cascade, $E_{i}<\epsilon_{\max }$, where $E_{i}$ is the central energy of each bin. We further only select bins for which the detection significance of the source is $S_{i}>2 \sigma$. 
The number of expected counts depends on the tested $B$-field hypothesis and on the intrinsic $\gamma$-ray spectrum. The latter can be determined from a power-law fit (including EBL absorption) to the energy bins for which the cascade contribution is negligible, i.e. $E_{i}>\epsilon_{\max }$. Example fits are shown in Figure 2 as red solid lines. The obtained best-fit parameters are then independent of the IGMF. The fit uncertainty can be incorporated into the likelihood by an efficiency term, $\beta_{i}$. The likelihood for this additional nuisance parameter can be assumed to follow a Gaussian, so that the total likelihood becomes

$$
\begin{aligned}
\mathcal{L}\left(\mu_{i}, \theta_{i} ; \alpha, \sigma_{i} \mid x_{i}, y_{i}\right)= & \left(2 \pi \sigma_{i}^{2}\right)^{-1 / 2} \exp \left(-\left(1-\beta_{i}\right)^{2} / 2 \sigma_{i}^{2}\right) \\
& \times \mathcal{L}\left(\beta_{i} \mu_{i}, b_{i} ; \alpha \mid x_{i}, y_{i}\right),
\end{aligned}
$$

where $\theta_{i}=\left(b_{i}, \beta_{i}\right)$ denotes the nuisance parameters and $\sigma_{i}$ is the relative theoretical flux uncertainty in the $i$ th energy bin from the full covariance matrix of the powerlaw fit (light-red shaded areas in Figure 2). We make the simplifying assumption that the best-fit intrinsic spectrum is equal to the input spectrum. Further systematic uncertainties can be implemented in a similar way.

Instead of generating many Monte Carlo realizations for the mock data sets, we make use of the so-called Asimov data set, for which $x$ and $y$ are equal to the expected number of counts (Cowan et al. 2011). Denoting the expected number of counts for the IGMF hypothesis under which the data are generated with $\mu^{D}$, the Asimov data set is $x_{i}=\mu_{i}^{D}+b_{i}$ and $y_{i}=b_{i} / \alpha$. For each source, we combine the likelihoods of all considered energy bins and find the profile likelihood by maximizing over the nuisance parameters. The likelihood ratio test (or test statistic, TS) is then

$$
\mathrm{TS}=-2 \sum_{\substack{i \\ E_{i}<\epsilon_{\max } \\ S_{i}>2 \sigma}} \ln \left(\frac{\mathcal{L}\left(\mu_{i}, \widehat{\theta}_{i}\left(\mu_{i}\right) ; \alpha, \sigma_{i} \mid \mu_{i}^{D}+b_{i}, b_{i} / \alpha\right)}{\mathcal{L}\left(\widehat{\mu}_{i}, \widehat{\theta}_{i} ; \alpha, \sigma_{i} \mid \mu_{i}^{D}+b_{i}, b_{i} / \alpha\right)}\right) .
$$

By virtue of the Asimov data set, the maximum likelihood estimators are simply $\widehat{\mu}_{i}=\mu_{i}^{D}$ and $\widehat{\theta}_{i}=(b, 1)$. In the numerator, the likelihood is maximized for fixed $\mu_{i}$ in terms of the background counts and efficiency to yield $\widehat{\theta}\left(\mu_{i}\right)$ (Rolke et al. 2005).

Applying Wilks' theorem, the test statistic should asymptotically follow a $\chi^{2}$ distribution with 2 degrees of freedom $\nu$ for the two model parameters $(B, \lambda)$. This allows us to convert the TS values into a significance $p_{\chi_{\nu}^{2}=2}$, with which we can exclude a magnetic field hypothesis for a given mock data set. To improve the sensitivity, the likelihoods of the different sources are combined by adding the TS values.

Since we do not know the IGMF morphology realized in nature, we generate mock data sets for specific scenarios of the IGMF strength and coherence length, yielding $\mu^{D}$ for each energy bin and source (see Section 5). The TS values are then computed with respect to the number of expected counts $\mu$ for all considered $(B, \lambda)$ values, which are extracted from the cascade simulations. We thus obtain the significances $p_{\chi_{\nu}^{2}=2}$ to rule out IGMF morphologies different from the one assumed in each scenario.

\section{RESULTS}

We generate mock data samples for three different representative IGMF configurations: $D_{1}=\left(B_{\mathrm{G}}=\right.$ $\left.10^{-15} ; \lambda_{\mathrm{Mpc}}=10^{-6}\right), D_{2}=\left(B_{\mathrm{G}}=10^{-16} ; \lambda_{\mathrm{Mpc}}=1\right)$, and $D_{3}=\left(B_{\mathrm{G}}=10^{-13} ; \lambda_{\mathrm{Mpc}}=10\right)$. The hypothesis $D_{1}$ corresponds to the case where the IGMF is of primordial origin (e.g. Durrer \& Neronov 2013) and is still allowed by the limits derived by Finke et al. (2015). The small values of $\lambda$ will suppress large deflection of the $e^{+} e^{-}$pairs. On the other hand, the values of $D_{3}$, close to the IGMF configuration suggested by observations of the diffuse $\gamma$-ray background (Chen et al. 2015b), will lead to large deflections, and most cascade photons will arrive with large time delays and outside $r_{80}$. The $D_{2}$ scenario corresponds to an intermediate case in terms of deflections and tests the hint for a nonzero IGMF deduced from evidence of pair halos in Fermi-LAT data (Chen et al. 2015a). Fields with such values of $\lambda$ could be generated by outflows from AGNs (e.g. Furlanetto \& Loeb 2001).

Figure 3 shows the possible limits in the $(B, \lambda)$ plane obtained from the observation of each source alone for $D_{j}, j=1,2,3$ and the fiducial set of model parameters. Both cuts on the angular separation and delay time are applied. The most constraining limits come from the simulated observation of B2 $0806+35$. The assumptions for the intrinsic spectrum are the most optimistic of our source sample as we extrapolate the 2FHL spectrum up to $E_{\tau=5} \sim 14 \mathrm{TeV}$, albeit the smallest ratio of $\mathrm{X}$ ray to radio flux (compare Table 1 ). This high-energy cutoff causes a plenitude of cascade photons in the energy range of CTA. Such an observation would lead to strong bounds on the IGMF, with possible exclusions beyond the $5 \sigma$ confidence level. On the other hand, for the highest-redshift source, and therefore smallest maximum energy $E_{\tau=5} \sim 4 \mathrm{TeV}$, no IGMF value can be ruled out. This is already obvious from Figure 2: the source is too faint below $\epsilon_{\max }$ to distinguish between the different IGMF scenarios. In the case of 1 ES $0229+200$ the fit uncertainties are large, and therefore no exclusions are possible if $\mathrm{T}_{\mathrm{obs}}=20$ hours. Doubling the observation time leads to mild exclusions, as visible in Figure 3. The two remaining sources give similar constraints at the $2 \sigma-3 \sigma$ level for $D_{1}$ and $D_{3}$. For $D_{2}$, the differences in the spectra are not pronounced enough to rule out IGMF configurations leading to either smaller or 
larger deflections. As expected, the limits are independent of the coherence length as long as $\lambda \gg D_{\text {IC }}$. The small structures in the exclusion plots can be explained with the intrinsic scatter of the ELMAG simulations.

We combine the likelihoods of the sources and reevaluate the exclusion regions (Figure 4, top panels). We exclude the source B2 $0806+35$, as we consider its cascade yield uncertain due to the extrapolation of the intrinsic spectrum by more than one order of magnitude and its small $F_{\mathrm{X}} / F_{\mathrm{R}}$ ratio. The limits considerably improve for all tested hypotheses. If the IGMF configurations close to $D_{3}$ are realized in nature, CTA observations could improve current limits (Finke et al. 2015) by two orders of magnitude. The $D_{1}$ case results in stringent upper bounds on the IGMF, and field strengths with $B_{\mathrm{G}} \gtrsim 10^{-14}$ can be excluded at high significance, independent of the coherence length. The reason is that most cascade photons still arrive within the containment radius and with small time delays, leading to a large excess at $\mathrm{GeV}$ energies. For higher IGMF strengths or coherence lengths, the excess decreases, which is incompatible with this particular mock data set. Therefore, upper limits on $B$ are obtained. This situation will always occur for IGMF scenarios that lead to minimal deflections of the cascade photons. For $D_{2}$, the combined likelihood leads to an exclusion of magnetic fields smaller than $10^{-17} \mathrm{G}$ at $3 \sigma$. Larger fields are at tension with the data at $1 \sigma-2 \sigma$. As for the single-source limits, the combined limits only show a dependence on the coherence length if $\lambda_{\mathrm{Mpc}} \lesssim 10^{-2}$.

Relaxing the cut on the maximum delay time has a strong impact on the sensitivity to the detection of cascade photons (Figure 4, bottom panels). Applying no cut on the maximum time delay at all is certainly an oversimplification due to finite AGN lifetimes, which are estimated to lie between $10^{6}$ and $10^{8} \mathrm{yr}$ (e.g., Parma et al. 2002). However, we do not expect a significant change if we would instead assume $\delta t<10^{8} \mathrm{yr}$, as even for the tested IGMF leading to the largest delays $\left(B_{\mathrm{G}}=10^{-11}, \lambda_{\mathrm{Mpc}}=100\right)$, a significant fraction of the cascade photons still arrive with smaller delays. Interestingly, comparing the $\delta t<10 \mathrm{yr}$ case to the case with no time cut, one sees that for configurations leading to small deflections (as in $D_{1}$ ), the projected limits worsen by two orders of magnitude. The reason is that more cascade photons reach the observer since the $\delta t$ cut is more stringent than the requirement $\delta \theta<r_{80}$ (see Figure 1). As a result, increasing $B$ or $\lambda$ will have a weaker effect on the spectra up to the point where $\delta \theta>r_{80}$. Consequently, configurations with $B_{\mathrm{G}} \lesssim 10^{-15}$ cannot be distinguished with high significance from a zero IGMF regardless of $\lambda$. This also explains the differences in $D_{2}$ and $D_{3}$. Only in the $D_{3}$ scenario does the applied cut on $\delta t$ lead to conservative limits (right panels of Figure 4). In this case, the time cut removes a large number of cascade photons so that the cascade bump is less pronounced even for small values of the magnetic field.

We further investigate the dependence of the projected limits on the chosen EBL model and energy of the spectral cutoff in Figure 5. In the top panels, the EBL model of Finke et al. (2010) is used instead of the photon density predicted by Domínguez et al. (2011), while $E_{\tau=5}$ is held constant. For the Finke et al. model, the limits are strengthened since the attenuation is slightly higher for sources with $z \gtrsim 0.1$. As the attenuation increases, so does the number distribution of the $e^{+} e^{-}$pairs, which is given in the steady-state approximation by

$$
N(\gamma)=|\dot{\gamma}|^{-1} \int_{\gamma}^{\infty} d \gamma^{\prime} Q\left(\gamma^{\prime}\right),
$$

where $\dot{\gamma}=c \gamma^{3} / D_{\text {IC }} \propto \gamma^{2}$ is the energy loss of the pairs due to IC scattering and $Q(\gamma)=\mathrm{d} N / \mathrm{d} E(1-\exp (-\tau))$ is the injection rate with $E=2 m_{e} c^{2} \gamma$. For hard intrinsic $\gamma$-ray spectra and since $\tau$ increases rapidly with energy, the integral of Eq. (7) will be almost independent of the lower integration bound and $N(\gamma)$ is dominated by $|\dot{\gamma}|^{-1}$, so that $N(\gamma) \propto \gamma^{-2}$. Thus, $N(\gamma)$ is dominated by low energy pairs (Tavecchio et al. 2011). For $\gamma=5 \times 10^{4}$ (corresponding to a $50 \mathrm{GeV} \gamma$-ray), $N(\gamma)$ is about $4 \%-$ $5 \%$ larger for the Finke et al. (2010) model.

Considering instead the EBL model of Domínguez et al. (2011) but lowering the maximum spectral energy to the value that corresponds to $\tau_{\max }=4$ decreases the sensitivity significantly (middle panels of Figure 5). The situation is reversed if we increase the maximum energy so that $\tau_{\max }=6$ (bottom panels of Figure 4$) .{ }^{1}$ In this case, the IGMF strength could be determined within one order of magnitude in the $D_{2}$ scenario. These findings underline the necessity that the intrinsic spectra need to extend to energies as high as possible in order to derive strong constraints on the IGMF.

We have also tested the dependence on the jet opening angle, and even a highly collimated jet with $\theta_{\mathrm{j}}=1^{\circ}$ has a negligible effect on the limits.

Throughout this work, we have assumed that the intrinsic blazar spectrum is given by a simple power law. In principle, the cascade component could be mimicked by features in the intrinsic blazar spectrum caused by, e.g., multiple $\gamma$-ray-emitting regions (e.g. Lefa et al. 2011a). However, the multiwavelength emission of EHBLs can also be successfully described with one emission zone under the assumption of very high

1 The corresponding energies are 10.6, 8.0, 7.5, and $5.6 \mathrm{TeV}$ for $1 \mathrm{ES} 0229+200, \mathrm{PG} 1218+304$, PMN J1548-2251, and 1RXS J023832.6-311658, respectively. 


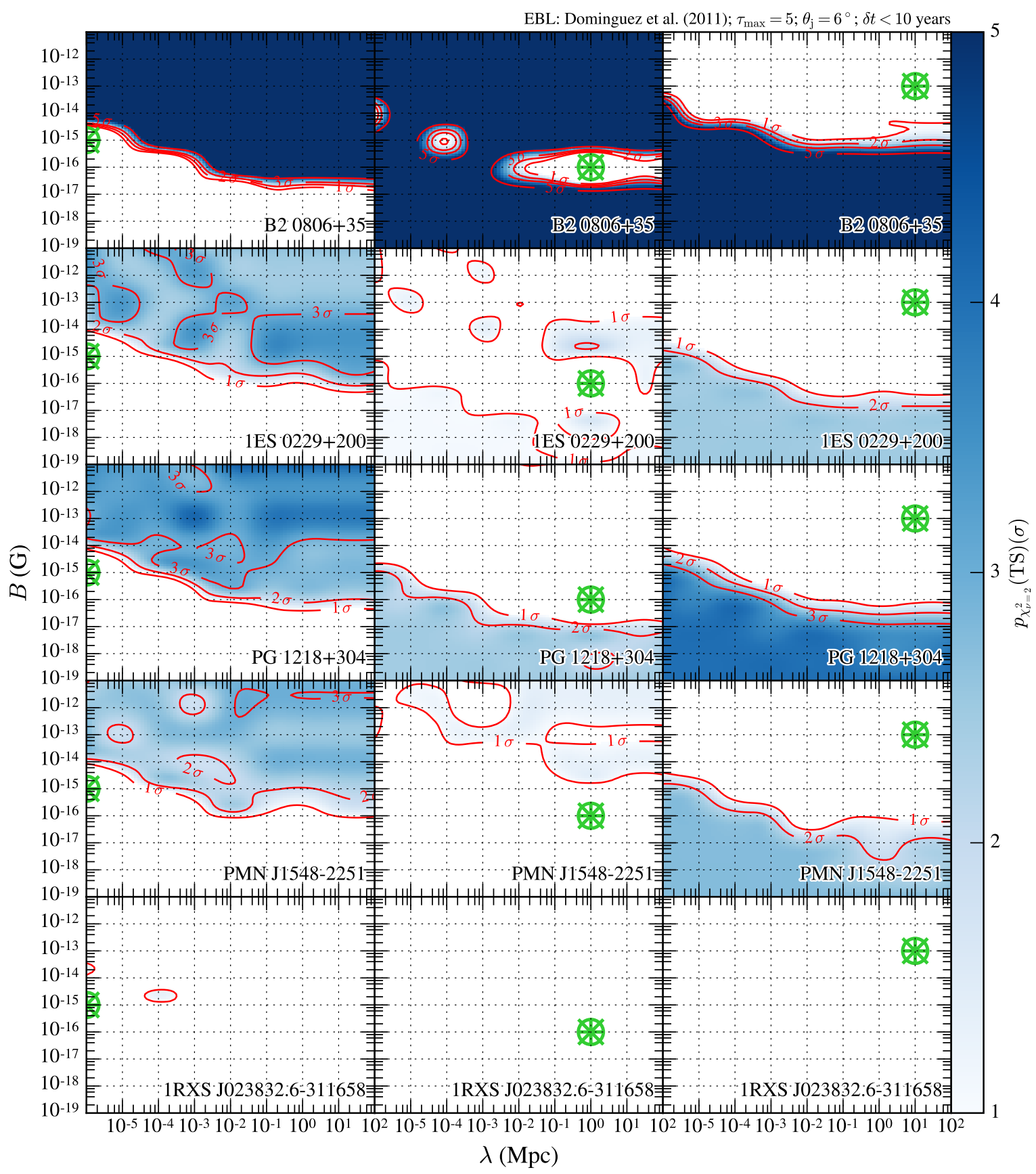

Figure 3. Exclusion regions for single-source observations (top to bottom) and different IGMF configurations (green markers). From left to right, the configurations are $\log _{10}\left(B_{\mathrm{G}}\right)=-15,-16,-13$ with coherence lengths $\log _{10}\left(\lambda_{\mathrm{Mpc}}\right)=-6,0,1$. The results on the parameter grid are interpolated with a bivariate spline.

minimal Lorentz factors of the underlying electron distributions or electron distributions with a Maxwellian shape (Katarzyński et al. 2006; Tavecchio et al. 2009; Kaufmann et al. 2011; Lefa et al. 2011b; Bonnoli et al. 2015). Furthermore, the sensitivity for the cascade has been derived here from a combined likelihood of several sources. It would appear highly contrived if the intrinsic spectra of multiple sources showed features at exactly the right energies where one expects the cascade component for a given IGMF.

\section{CONCLUSIONS}

Utilizing a standard likelihood ratio test, future observations with CTA of a small number of certain HBLs will yield strong constraints of the IGMF. Especially HBLs with hard spectra that extend to energies corresponding to an optical depth of $\tau \gtrsim 5$ are particularly well suited since a large amount of energy will be repro- 

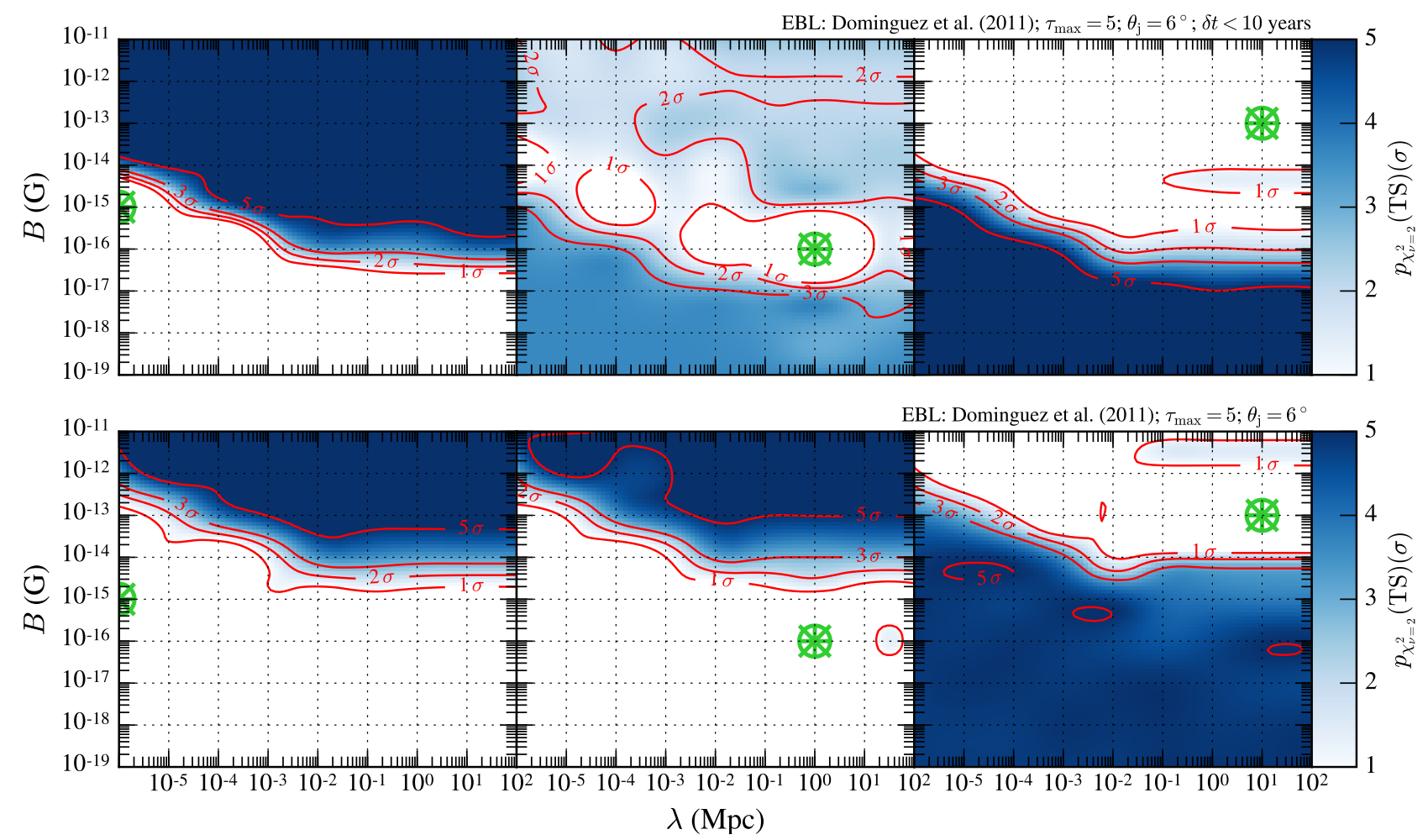

Figure 4. Exclusion plots from a combined likelihood of all blazars except B2 0806+35 for three tested IGMF configurations. Top: limits with a maximum time delay $\delta t<10$ years. Bottom: limits without any restrictions on the delay of the cascade photons.

cessed in the electromagnetic cascade. The large energy range covered with CTA makes it possible to probe the EBL cutoff (Mazin et al. 2013) and to ensure the absence of an intrinsic spectral break. Simultaneously, one can search for cascade photons at the low-energy end of the spectra. Throughout this article, we have assumed the "Array E" configuration of CTA (Bernlöhr et al. 2013). The final constraints derived from real data will depend on the actually realized configuration and data analysis.

Nevertheless, the (non)observation of a cascade excess in CTA spectra will allow us to limit an IGMF with a high (low) field strength depending on the actual IGMF morphology realized in nature, the maximum emitted $\gamma$-ray energies, and the duty cycles of the considered sources. CTA observations should be able to either confirm or rule out evidence of a nonzero IGMF (e.g. Essey et al. 2011; Chen et al. 2015a,b) and improve current limits on its field strength and coherence length by orders of magnitude (e.g. Taylor et al. 2011; Arlen et al. 2014; Finke et al. 2015). Especially if IGMF configurations are realized in nature that lead to either strong or very small deflections of the $e^{+} e^{-}$pairs, it will be possible to rule out large fractions of the IGMF parameter space. Magnetic fields of the order of $B \sim 10^{-16} \mathrm{G}$ with coherence lengths $\lambda \sim 1 \mathrm{Mpc}$ will be more difficult to constrain as the cascade radiation will only lead to a slight excess over the primary $\gamma$-ray emission.
CTA observations will in general not be able to distinguish between a primordial and astrophysical origin of the IGMF. Furthermore, the sensitivity estimates depend strongly on the assumed cutoff energy of the spectra. Spectra extending only up to energies so that $\tau_{\max }=4$ will not generate sufficient cascade radiation to constrain the IGMF. If, on the other hand, the spectra reach very high energies with optical depths $\tau \sim 6$, primordial IGMF scenarios could be ruled out given that the coherence length is $\lambda \gtrsim 0.1 \mathrm{Mpc}$. The projected limits also strongly depend on the assumed $\gamma$-ray activity time of the AGN. The maximum allowed delay time of cascade photons is degenerate with the IGMF strength, and small values of $\delta t$ only yield conservative limits if small values of values of the IGMF strength are to be constrained. It should be noted that the cell-like morphology of the IGMF adopted in the ELMAG code neglects the dependence of the limits on the actual IGMF power spectrum (Caprini \& Gabici 2015). Especially for red power spectra, the cell-like assumption breaks down and lower limits on the IGMF have to be relaxed.

We have only used photons arriving within the $80 \%$ containment radius of the PSF. In future work, the analysis should be extended to incorporate the extended pair-halo emission. This will add further information to the likelihood and will make it easier to distinguish between IGMF scenarios. Interestingly, due to the time 

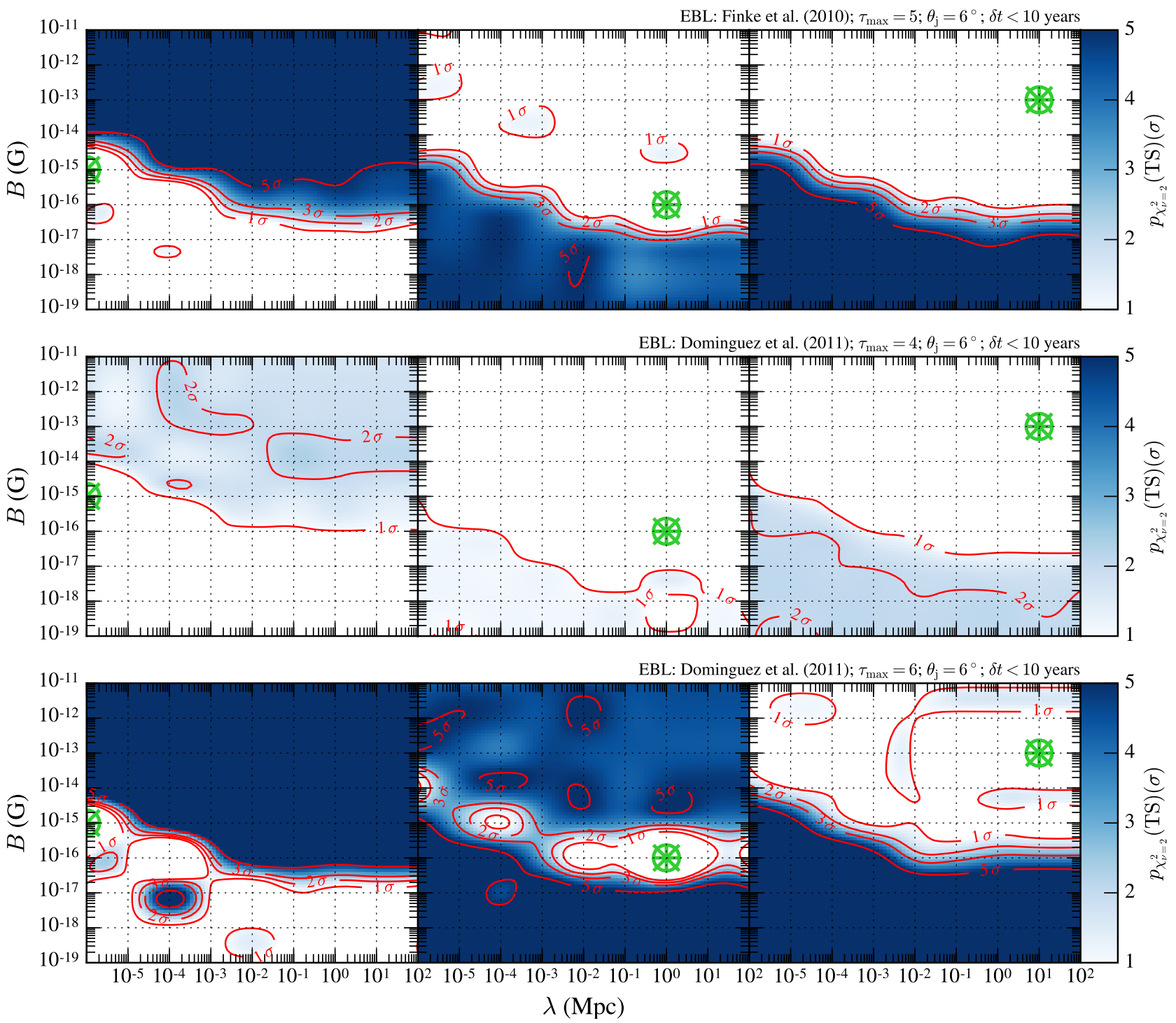

Figure 5. Same as Figure 4 with $\delta t<10 \mathrm{yr}$ but for the Finke et al. (2010) EBL model and $\tau_{\max }=5$ (top), and for the Domínguez et al. (2011) EBL model with $\tau_{\max }=4$ (middle) and $\tau_{\max }=6$ (bottom).

delay of the cascade photons, such halos could still be present even if the source already ceased its activity (Neronov et al. 2010; Inoue et al. 2011) and could be searched for the envisaged CTA extragalactic survey.

\section{ACKNOWLEDGEMENTS}

This paper has gone through internal review by the CTA Consortium. The authors would like to thank Anthony Brown, Jonathan Biteau, Matteo Cerruti, Michele Doro, Susumu Inoue, Kohta Murase, Elisabete de Gouveia Dal Pino, Vitor de Souza, and especially Alberto Domínguez for discussions and comments on the manuscript. J.C. is a Wallenberg Academy Fellow.

Software: ELMAG (Kachelrieß et al. 2012)

\section{REFERENCES}

Abramowski, A., et al. 2013, A\&A, 552, A118

-. 2014, A\&A, 562, A145

Acero, F., et al. 2015, ApJS, 218, 23

Ackermann, M., Ajello, M., Atwood, W. B., et al. 2015, ApJ, 810,14

—. 2016, ApJS, 222, 5
Actis, M., Agnetta, G., Aharonian, F., et al. 2011, Experimental Astronomy, 32, 193

Ade, P. A. R., et al. 2015, ArXiv e-prints, arXiv:1502.01594

Aharonian, F., et al. 2006, A\&A, 457, 899

-. 2007, A\&A, 475, L9

Aharonian, F. A., Coppi, P. S., \& Voelk, H. J. 1994, ApJL, 423, L5 
Archambault, S., et al. 2014, ApJL, 785, L16

—. 2016, ArXiv e-prints, arXiv:1603.02410

Arlen, T. C., Vassilev, V. V., Weisgarber, T., Wakely, S. P., \& Yusef Shafi, S. 2014, ApJ, 796, 18

Bernlöhr, K., Barnacka, A., Becherini, Y., et al. 2013, Astroparticle Physics, 43, 171

Bertone, S., Vogt, C., \& Enßlin, T. 2006, MNRAS, 370, 319

Blasi, P., Burles, S., \& Olinto, A. V. 1999, ApJL, 514, L79

Bonnoli, G., Ghisellini, G., Foschini, L., Tavecchio, F., \& Ghirlanda, G. 2011, MNRAS, 410, 368

Bonnoli, G., Tavecchio, F., Ghisellini, G., \& Sbarrato, T. 2015, MNRAS, 451, 611

Broderick, A. E., Chang, P., \& Pfrommer, C. 2012, ApJ, 752, 22

Caprini, C., \& Gabici, S. 2015, PhRvD, 91, 123514

Chen, W., Buckley, J. H., \& Ferrer, F. 2015a, Physical Review Letters, 115, 211103

Chen, W., Chowdhury, B. D., Ferrer, F., Tashiro, H., \& Vachaspati, T. 2015b, MNRAS, 450, 3371

Costamante, L., Ghisellini, G., Giommi, P., et al. 2001, A\&A, 371,512

Cowan, G., Cranmer, K., Gross, E., \& Vitells, O. 2011, European Physical Journal C, 71, 1554

CTA Consortium. 2016, in preparation.

Dai, Z. G., Zhang, B., Gou, L. J., Mészáros, P., \& Waxman, E. 2002, ApJL, 580, L7

Daly, R. A., \& Loeb, A. 1990, ApJ, 364, 451

Dermer, C. D., Cavadini, M., Razzaque, S., et al. 2011, ApJL, 733, L21+

Dolag, K., Grasso, D., Springel, V., \& Tkachev, I. 2005, JCAP, 1,9

Dolag, K., Kachelrieß, M., Ostapchenko, S., \& Tomàs, R. 2009, ApJ, 703, 1078

Dolag, K., Kachelriess, M., Ostapchenko, S., \& Tomàs, R. 2011, ApJL, 727, L4

Domínguez, A., \& Ajello, M. 2015, ApJL, 813, L34

Domínguez, A., Primack, J. R., Rosario, D. J., et al. 2011, MNRAS, 410, 2556

Durrer, R., \& Neronov, A. 2013, A\&A Rv, 21, 62

Dwek, E., \& Krennrich, F. 2013, Astroparticle Physics, 43, 112

Elyiv, A., Neronov, A., \& Semikoz, D. V. 2009, PhRvD, 80, 023010

Ensslin, T. A., Biermann, P. L., Kronberg, P. P., \& Wu, X.-P. 1997, ApJ, 477, 560

Essey, W., Ando, S., \& Kusenko, A. 2011, Astroparticle Physics, 35,135

Finke, J. D., Razzaque, S., \& Dermer, C. D. 2010, ApJ, 712, 238

Finke, J. D., Reyes, L. C., Georganopoulos, M., et al. 2015, ApJ, 814,20

Furlanetto, S. R., \& Loeb, A. 2001, ApJ, 556, 619

Furniss, A., Williams, D. A., Danforth, C., et al. 2013, ApJL, 768, L31

Gould, R. J., \& Schréder, G. P. 1967a, Physical Review, 155, 1408

—. 1967b, Physical Review, 155, 1404

Grasso, D., \& Rubinstein, H. R. 2001, PhR, 348, 163

Huan, H., Weisgarber, T., Arlen, T., \& Wakely, S. P. 2011, ApJL, 735, L28

Inoue, S., Takahashi, K., Mori, M., \& Ichiki, K. 2011, in AGN Physics in the CTA Era (AGN 2011), 32
Jelley, J. V. 1966, Physical Review Letters, 16, 479

Kachelrieß, M., Ostapchenko, S., \& Tomàs, R. 2012, Computer Physics Communications, 183, 1036

Katarzyński, K., Ghisellini, G., Tavecchio, F., Gracia, J., \& Maraschi, L. 2006, MNRAS, 368, L52

Kaufmann, S., Wagner, S. J., Tibolla, O., \& Hauser, M. 2011, A\&A, 534, A130

Kulsrud, R. M., \& Zweibel, E. G. 2008, Reports on Progress in Physics, 71, 046901

Lefa, E., Aharonian, F. A., \& Rieger, F. M. 2011a, ApJL, 743, L19

Lefa, E., Rieger, F. M., \& Aharonian, F. 2011b, ApJ, 740, 64

Li, T.-P., \& Ma, Y.-Q. 1983, ApJ, 272, 317

Madhavan, A. S. 2013, ArXiv e-prints, arXiv:1307.7051

Mazin, D., Raue, M., Behera, B., et al. 2013, Astroparticle Physics, 43, 241

Menzler, U., \& Schlickeiser, R. 2015, MNRAS, 448, 3405

Meyer, M., \& Conrad, J. 2014, JCAP, 12, 016

Meyer, M., Raue, M., Mazin, D., \& Horns, D. 2012, A\&A, 542, A59

Murase, K., Takahashi, K., Inoue, S., Ichiki, K., \& Nagataki, S. 2008, ApJL, 686, L67

Muriel, H., Donzelli, C., Rovero, A. C., \& Pichel, A. 2015, A\&A, 574, A101

Neronov, A., Semikoz, D., Kachelriess, M., Ostapchenko, S., \& Elyiv, A. 2010, ApJL, 719, L130

Neronov, A., \& Semikoz, D. V. 2009, PhRvD, 80, 123012

Neronov, A., \& Vovk, I. 2010, Science, 328, 73

Nikishov, A. I. 1962, Sov. Phys. JETP, 14, 393

Parma, P., Murgia, M., de Ruiter, H. R., \& Fanti, R. 2002, NewAR, 46, 313

Plaga, R. 1995, Nature, 374, 430

Plotkin, R. M., Anderson, S. F., Brandt, W. N., et al. 2010, AJ, 139, 390

Protheroe, R. J., \& Stanev, T. 1993, MNRAS, 264, 191

Pshirkov, M. S., Tinyakov, P. G., \& Urban, F. R. 2015, ArXiv e-prints, arXiv:1504.06546

Rees, M. J. 1987, QJRAS, 28, 197

Rolke, W. A., López, A. M., \& Conrad, J. 2005, Nuclear Instruments and Methods in Physics Research A, 551, 493

Shaw, M. S., Romani, R. W., Cotter, G., et al. 2013, ApJ, 764, 135

Sigl, G., Miniati, F., \& Enßlin, T. A. 2004, Nuclear Physics B Proceedings Supplements, 136, 224

Sironi, L., \& Giannios, D. 2014, ApJ, 787, 49

Takahashi, K., Murase, K., Ichiki, K., Inoue, S., \& Nagataki, S. 2008, ApJL, 687, L5

Tashiro, H., \& Vachaspati, T. 2013, PhRvD, 87, 123527

Tavecchio, F., Ghisellini, G., Bonnoli, G., \& Foschini, L. 2011, MNRAS, 414, 3566

Tavecchio, F., Ghisellini, G., Ghirlanda, G., et al. 2009, MNRAS, 399, L59

Taylor, A. M., Vovk, I., \& Neronov, A. 2011, A\&A, 529, A144

Widrow, L. M. 2002, Reviews of Modern Physics, 74, 775 